\section{Primär pulmonale Manifestation einer extramedullären akuten myeloischen Leukämie}

A. Florschütz', H.-J. Schumann², C. Erikson³, M. Zugehör ${ }^{1}$, W. Rosahl ${ }^{1}$, J. Schreiber ${ }^{1}$

Städtisches Klinikum Dessau, Akademisches Lehrkrankenhaus der Martin-Luther-Universität Halle-Wittenberg

${ }^{1}$ Klinik für Innere Medizin (Chefarzt: Dr. med. W. Rosahl), Abteilung für Hämatologie/Onkologie und Abteilung für Pneumologie

2 Institut für Pathologie (Chefarzt: Dr. med. J. J. Knolle)

${ }^{3}$ Institut für Radiologie und Röntgendiagnostik (Chefarzt: PD Dr. med. H. König)

\section{Fallbeschreibung}

Zusammenfassung: Es wird der Fall einer 49-jährigen Patientin mit einer primär pulmonalen Manifestation einer akuten myeloischen Leukämie ohne sicheren leukämischen Nachweis beschrieben. Die Diagnosestellung erfolgte durch den Nachweis leukämischer Blasten in der bronchoalveolären Lavage. Eine Chemotherapie nach dem TAD-VP-Protokoll führte zu einer partiellen Remission. Im weiteren Verlauf kam es jedoch zu einem therapierefraktären, nunmehr generalisierten Frührezidiv, an dem die Patientin verstarb. In der recherchierten Literatur ist dies die erste Beschreibung einer primären, isolierten extramedullären Manifestation einer AML in den Lungen.

Primary extra-medullary manifestation of a acute myeloid leukemia in the lungs - a case report: We report on a 49 year old female with primary extra-medullary manifestation of a acute myeloid leukemia in the lungs without leukemic signs. The disease was diagnosed by detection of leukemic blast cells in bronchoalveolar lavage. Chemotherapy with the TAD-VP-scheme resulted in partiell remission. The patient died in systemic early relapse. To our knowledge this is the first description of primary isolated extra-medullary manifestation of a acute myeloid leukemia in the lungs.

\section{Einleitung}

Die akute myeloische Leukämie (AML) ist eine seltene maligne Erkrankung, der die unkontrollierte klonale Proliferation von myeloischen Vorläuferzellen zugrunde liegt. Typischerweise wird die Diagnose über den Nachweis der Leukämiezellen im peripheren Blut und im Knochenmark gestellt. Die ausschließlich extramedulläre Manifestation wird als Chlorom oder granulozytisches Sarkom bezeichnet und ist oft schwierig zu diagnostizieren [1]. Meist sind die Lymphknoten, die Haut oder das Nervensystem beteiligt. Die Prognose ist bei extramedullärer Manifestation der AML meist schlecht.

Leukämische Infiltrationen der Lunge können im Verlauf der Erkrankung auftreten. Eine pulmonale Erstmanifestation einer AML ist eine Rarität [2-4]. In der recherchierten Literatur haben wir keinen Fall einer isolierten pulmonalen Erstmanifestation gefunden.

Pneumologie 2001; 55: $302-305$

(c) Georg Thieme Verlag Stuttgart · New York ISSN 0934-8387 49-jährige Patientin, seit 4 Wochen rasche Verschlechterung des Allgemeinzustandes mit progredienter Dyspnoe, unproduktivem Husten und Gewichtsverlust, kein Fieber oder Nachtschweiß. Eine ambulante Antibiotikatherapie war erfolglos.

\section{Klinische Untersuchung:}

Reduzierter Allgemein- und Ernährungszustand, blasses Hautcolorit, verschärftes Atemgeräusch über beiden Lungen mit vereinzelten feinblasigen Rasselgeräuschen. Herz, Gefäßstatus und Abdomen unauffällig, insbesondere keine Hepatosplenomegalie, keine peripheren Lymphknotenschwellungen.

\section{Laborbefunde:}

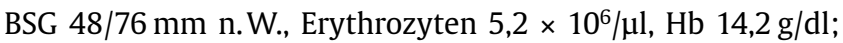
MCV 91,7 fl; Thrombozyten $419 \times 10^{3} / \mu$; Leukozyten $18,4 \times$ $10^{3} / \mu \mathrm{l}$ (75\% Segmentkernige, $1 \%$ Stabkernige, 10\% Lymphozyten, 2\% Lymphoidzellen, 9\% Monozyten, 2\% Basophile, 1\% Eosinophile).

LDH 8,0 $\mu \mathrm{mol} / \mathrm{l}$; CrP 126,7 mg/l; Ges.Eiweiß 69,4 g/l; Serumelektrophorese mit 43,9\% Albumin; 8,1\% Alpha1; 19,5,\% Alpha2; 12,4\% Beta, 16,1\% Gamma.

Im Normbereich: Gerinnung, Elektrolyte, Kreatinin, Harnstoff, GOT, GPT, GGT, Bilirubin, AP, TSH, Fe, Lipase.

Blutgasanalyse: $\mathrm{pH}$ 7,45; StBi 23,4 mmol/l; $\mathrm{BE}-2 \mathrm{mmol} / \mathrm{l}$; $\mathrm{paCO}_{2} 43 \mathrm{~mm} \mathrm{Hg}$; $\mathrm{paO}_{2} 69 \mathrm{~mm} \mathrm{Hg} ; \mathrm{O}_{2}$-Sättigung $91 \%$.

Autoantikörper: ANA, AMA, c- und p-ANCA negativ.

Infektionsserologie: kein Anhalt für eine floride oder abgelaufene Infektion mit HIV, CMV, HSV, Mycoplasmen, Candida oder Aspergillus. Blut- und Urinkulturen: kein Keimnachweis.

Tuberkulintest (10 TE): negativ.

EKG und Echokardiographie: unauffällig.

Sonographie: Leber, Milz, Nieren, Pankreas, biliäres System und paraaortaler Raum unauffällig. Kein Nachweis von Lymphomen, kein Pleuraerguss.

Gastroskopie und Koloskopie: unauffällig. 


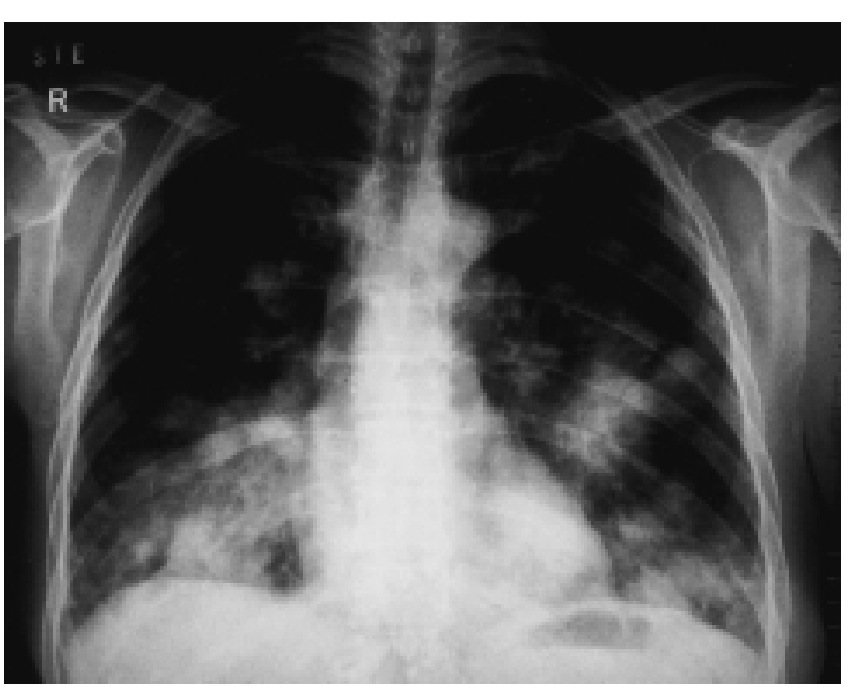

Abb. 1 Röntgen des Thorax zum Aufnahmezeitpunkt.

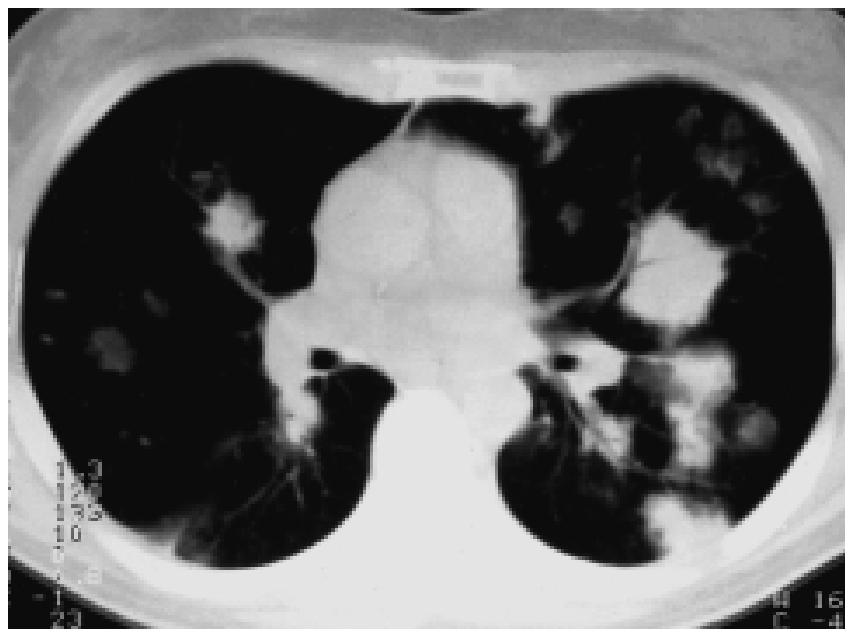

Abb. 2 Computertomographie des Thorax zum Aufnahmezeitpunkt.

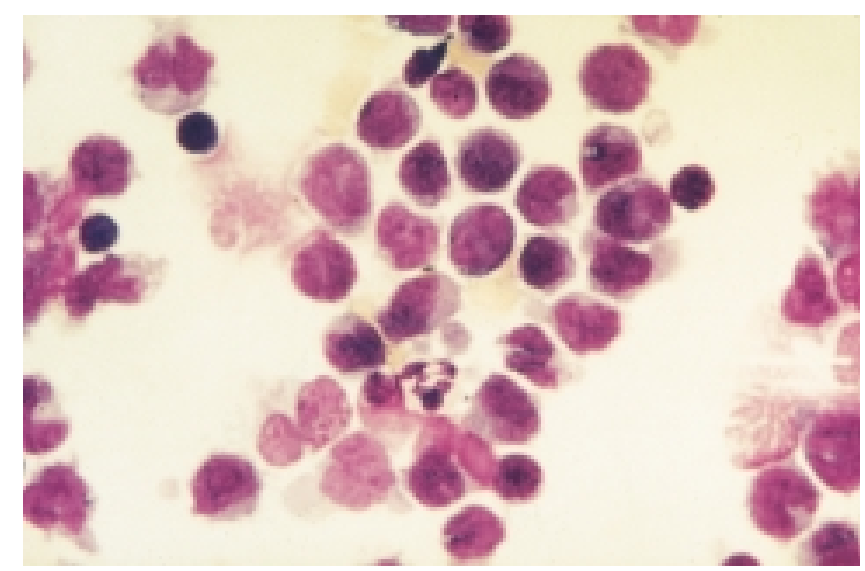

Abb. 3 Bronchoalveoläre Lavage mit Nachweis von atypischen monozytoiden Zellen.
Röntgen-Thorax (Abb.1): beidseitig multiple, inhomogene teils konfluierende rundliche Verdichtungen.

CT-Thorax (Abb. 2): in beiden Lungen Nachweis multipler, z.T. konfluierender weichteildichter Raumforderungen.

Bodypletysmographie: mittelgradige Restriktion, keine Obstruktion.

Bronchoskopie: geringe Zeichen einer floriden Bronchitis, endobronchial kein Tumor,

Mikrobiologie: im Bronchialsekret und in der BAL kein Nachweis einer relevanten Flora.

Bronchoalveoläre Lavage (BAL) (Abb. 3): Gesamtzellzahl 1350 Mpt/l (12\% Lymphozyten, 7\% Neutrophile, 2\% Speicherzellen, $2 \%$ Schaumzellen, $77 \%$ monozytoide Zellen).

Die atypischen monozytoiden Zellen haben blastären Charakter. Schwach positive Reaktion auf Esterase, keine Reaktion mit Peroxidase oder PAS, dringender Verdacht auf eine leukämische Infiltration durch eine AML.

Immunphänotypisierung: In der BAL Nachweis einer etwa 70\%-Population von CD33, CD14, CD45, HLA-DR und CD7 positiven und CD34, CD13, CD117, CD10, CD20 und CDw65 negativen Zellen.

Histologie von transbronchialen Bioptaten: ausgeprägte Makrophagenreaktion in den Alveolen, leichte alveolarseptale Fibrose sowie eine vorwiegend intramurale, in geringem Maße aber auch intraalveoläre mononukleäre Infiltration. Kein Anhalt für eine Vasculitis. Keine granulomatöse Reaktion.

Immunphänotypisierung Blut: Nachweis einer Population (etwa 15\%) von myeloischen Zellen (CD 33, CD14, CD117, HLADR und CD7 positiv, sowie CD34, CD13 und CDw65 negativ).

Knochenmarkzytologie: geringe reaktive Veränderungen, kein sicherer Anhalt für Knochenmarkinfiltration durch eine AML.

Immunphänotypisierung Knochenmark: Bei weitgehend normaler Verteilung fallen etwa 9\% myelomonozytäre Zellen (positiv mit CD33, CD14 und HLA-DR) mit Koexpression von CD7 auf.

Knochenmarkhistologie: regeneratorisches Knochenmark, keine Leukose.

\section{Therapie und Verlauf}

Auf Grundlage der zytologischen Befunde und der Immunphänotypisierung der BAL und nach Ausschluss wichtiger Differenzialdiagnosen wurde die Diagnose einer primär extramedullären Manifestation einer akuten myeolischen Leukämie gestellt. Bei rasch progredienter Verschlechterung des Zustandes der Patientin wurde auf weitere Versuche der histologischen Sicherung verzichtet und eine Chemotherapie nach dem TAD-VP-Protokoll (Thioguanin, Cytarabin, Daunorubicin, Vincristin und Prednisolon) durchgeführt. Es kam innerhalb einer Woche zu einer deutlichen klinischen Besse- 
rung und innerhalb von 4 Wochen $\mathrm{zu}$ einer ausgeprägten Regredienz der pulmonalen Infiltrate.

Eine erneute Bronchoskopie zeigte in der BAL weiterhin die bekannte Zellpopulation mit dem bekannten immunphänotypischen Expressionsmuster. Wegen dieser Befunde wurde die Therapie intensiviert und die Patientin nach dem HAMProtokoll (hochdosiertes Cytarabin und Mitoxantron) behandelt. Dies führte zu einer weiteren Regredienz der pulmonalen Infiltrate.

Wir planten deshalb eine weitere HAM-Therapie und bei Fehlen eines HLA-identischen Familienspenders eine Hochdosistherapie mit autologer Stammzelltransplantation.

Kurz vor der Realisierung dieser Therapie verschlechterte sich der Allgemeinzustand der Patientin rapide. Die pulmonalen Infiltrate nahmen deutlich zu. Auch im Blut und im Knochenmark waren jetzt leukämische Zellen sicher nachweisbar. Es wurde eine zytoreduktive Vorphasentherapie mit Cytarabin als Dauerinfusion durchgeführt. Der Zustand der Patientin stabilisierte sich nicht. Trotz intensivmedizinischer Maßnahmen verstarb die Patientin bei floridem Tumorprogress an einem Multiorganversagen.

Eine Autopsie wies multiple z.T. metastatisch anmutende Infiltrate im Knochenmark, den Lungen aber auch in der Leber, den Lymphknoten, im Pankreas, in den Nieren und der Milz durch leukämische Blasten nach und bestätigte damit die klinische Diagnose.

\section{Diskussion}

Progrediente beidseitige pulmonale Infiltrationen erfordern eine vielfältige oft schwierige Differenzialdiagnose und nur sehr selten wird es sich dabei um leukämische Infiltrate handeln. Pulmonale Symptome treten bei einer AML infolge einer direkten Infiltration des Lungenparenchyms oder infolge einer Leukostase meist erst dann auf, wenn die Blastenzahlen im peripheren Blut $100000 / \mathrm{mm}^{3}$ überschreiten [6]. Bei Patienten mit akuten oder chronischen Leukämien werden Infiltrationen der Lungen entweder durch die Erkrankung selbst hervorgerufen oder sie sind Folge der Behandlung. Dabei sind diffuse oder lokalisierte leukämische Infiltrate, eine alveoläre Hämorrhagie, bakterielle, virale und mykotische Infektionen, opportunistische Infektionen, das pulmonale Leukostasesyndrom und toxische Lungenschädigungen durch Zytostatika abzugrenzen [5].

In der Differenzialdiagnostik von infiltrativen Lungenerkrankungen hat die Bronchoskopie mit bronchoalveolärer Lavage nach Möglichkeit auch mit transbronchialer Lungenbiopsie einen hohen Stellenwert. Wenn es bei der BAL nicht zu einer Blutbeimengung mit Leukämiezellen aus der systemischen Zirkulation gekommen ist, beweist der Nachweis von Leukämiezellen in der BAL die leukämische Infiltration der Lunge auch ohne histologische Verifizierung [5]. Neben der zytologischen Beurteilung der Lavage ist auch eine immunphänotypische Charakterisierung der Zellen wie bei leukämischen Populationen aus dem Blut oder Knochenmark möglich. Bei unserer Patientin waren in der BAL fast ausschließlich Myeloblasten nachweisbar.
Eine weitere Analyse im Blut und im Knochenmark ist bei Verdacht auf eine Leukämie obligat. In diesen Untersuchungsmaterialien konnte bei unserer Patientin eine leukämische Zellpopulation initial nicht nachgewiesen werden, wobei per definitionem bei Nachweis von $>20 \%$ Parablasten im Blut und/oder Knochenmark eine Leukämie besteht [7]. Hiermit ist einerseits die primär extramedulläre Erkrankung mit pulmonaler myeloblastärer Infiltration belegt, andererseits berechtigt es auch zu der Einschätzung, dass die Leukämiezellen in der BAL nicht aus der systemischen Zirkulation stammen können. Über eine derartige primär ausschließlich pulmonale Manifestation wurde in der von uns recherchierten Literatur bisher nicht berichtet.

Extramedulläre Infiltrationen können im Verlauf von akuten und chronischen Leukämien verschiedene Organe betreffen. In Autopsiestudien wurden extramedulläre Manifestationen von akuten Leukämien bei 24-66\% der Patienten beschrieben [8]. Die Prognose ist beim Auftreten von extramedullären Infiltrationen meist schlecht.

Die akuten myeloischen Leukämien werden morphologisch in verschiedene FAB-Typen klassifiziert [9]. Hierbei scheint eine extramedulläre Infiltration bei myelomonozytären (FAB M4) bzw. monozytären (FAB M5) akuten Leukämien häufiger aufzutreten $[4,6]$.

Bei der immunphänotypischen Untersuchung von Leukämiezellen wird bei der AML neben CD45, CD33, CD14, CD13, CD117, CDw65 zur Charakterisierung des myeloischen Ursprungs der Leukämiezelle auch nach einer Koexpression von lymphatischen Oberflächenmarkern gesucht (z. B. CD2, CD7, CD56, CD19) [10]. Bei unserer Patientin lag eine CD7-positive myeloische Zellpopulation vor. Die CD7 positive AML wurde als eine Entität charakterisiert, welche ein geringes Therapieansprechen und eine schlechtere Prognose hat [11]. In neueren Arbeiten wird insbesondere die CD7/CD56 positive AML als Leukämieform mit schlechter Prognose und häufiger extramedullärer Beteiligung beschrieben $[12,13]$.

Bei Nachweis von leukämischen Infiltraten ist die Therapie des malignen Leidens entsprechend den geltenden Empfehlungen unverzüglich nach Diagnosestellung einzuleiten. Die durchgeführte Chemotherapie führte initial zu einer schnellen Besserung, nicht aber zu der angestrebten kompletten Remission. Der bei extramedullärem Befall meist schlechtere Verlauf der akuten Leukämie bestätigte sich auch bei unserer Patientin. Bei progredienter pulmonaler und leukämischer Manifestation im Frührezidiv belegte dann die Autopsie sicher die anfängliche Diagnose. 


\section{Literatur}

${ }^{1}$ Menasce LP, Banerjee SS, Beckett E, Harris E. Extramedullary myeloid tumor is often misdiagnosed: a Study of 26 cases. Histopathology 1999; 34: 391 - 398

2 Byrd JC, Edenfield WJ, Shields DJ, Dawson NA. Extramedullary myeloid cell tumors in acute nonlymphocytic leukemia. J Clin Oncol 1995; 13: 1800-1816

${ }^{3}$ Meidenbauer N, Schlake G, Bross K, Andreesen R. Symptomatische leukämische Infiltration der Lunge als Komplikation einer akuten myeloischen Leukämie. Dtsch med Wschr 1998; 123: $110-113$

${ }^{4}$ Bhatia M, Coppage L. Dyspnoea, nonproductive cough and blasts on periphereal smear. Chest 1995; 107: 269-270

${ }^{5}$ Teschler H. Leukämien mit Lungenbeteiligung. In: Konietzko, N., Wendel, H., Wiesner, B. (Hrsg.) Erkrankungen der Lunge. Berlin, New York: De Gruyter, 1995: 400-401

${ }^{6}$ Kovalski R, Hansen-Flaschen J, Lodato RF, Pietra GG. Localized leukemic pulmonary infiltrates: Diagnosis by bronchoscopy and resolution with therapy. Chest 1990; 97: 674 - 678

${ }^{7}$ Harris NL, Jaffe ES, Diebold J, Flandrin G, Müller-Hermelink HK, Vardiman J, Lister TA, Bloomfield CD. World Health Organisation classification of neoplastic diseases of the hematopoetic and lymphoid tissues: report of the clinical advisor committee meeting-Airlie House Virginia November 1997. J Clin Oncol 1999; 17: 3835

${ }^{8}$ Barcos M, Warren L, Gomez GA, Han T, Freeman A, Preisler H, Henderson E. An autopsy study of 1206 acute and chronic leukemias 1958-1982. Cancer 1987; 60: 827-837

${ }^{9}$ Second MIC Cooperative Study Group. Morphologic, immunologic and cytogenetic (MIC) working classification of the acute myelogenous leukemias. Cancer Genetics Cytogenet 1988; 30: $1-15$

10 Jennings CD, Foon KA. Recent advances in flow cytometry: application to the diagnosis of hematologic malignancy. Blood 1997; 90: $2863-2892$

${ }^{11}$ Kita K, Miwa H, Nakase K, Kawakami K, Kobayashi T, Shirakawa S, Tanaka I, Ohta C, Tsutani H, Oguma S. Clinical importance of the CD7 expression in acute myelocytic leukemia. The Japan Cooperative Group of Leukemia/Lymphoma. Blood 1993; 81: 2399 - 2405

12 Kahl C, Florschütz A, Leuner S, Arland M, Jannsen JWG, Franke A, Höffkes HG. CD7/56 positive Acute Myelogenous Leukemia. Laboratory Hematology 1999; 5: 115-120

${ }^{13}$ Suzuki R, Yamamoto K, Setro M, Kagami Y, Ogura M, Yatabe Y, Suchi T, Kodera Y, Morishima Y, Takahashi T, Saito H, Ueda R, Nakamura S. CD7 and CD56 + myeloid/natural killer cell-type precursor acute leukemia: a distinct hematological disease entity. Blood 1997; 90: 2417 -2428

\section{Dr. med. A. Florschütz}

Städtisches Klinikum Dessau

Klinik für Innere Medizin

Abteilung für Hämatologie/Onkologie

Auenweg 38

06847 Dessau

E-mail: SKD.AFlorschuetz@t-online.de 